\title{
Challenges in identifying antibiotic resistance targets for point-of-care diagnostics in general practice
}

\author{
Konstantinos Mitsakakis ${ }^{1,2}$, Wendy E Kaman ${ }^{3}$, Gijs Elshout ${ }^{4}$, Mara Specht ${ }^{1}$ \& John P Hays J $^{*}$ \\ ${ }^{1}$ Hahn-Schickard, Georges-Koehler-Allee 103, 79110 Freiburg, Germany \\ ${ }^{2}$ Laboratory for MEMS Applications, IMTEK - Department of Microsystems Engineering, University of Freiburg, \\ Georges-Koehler-Allee 103, 79110 Freiburg, Germany \\ ${ }^{3}$ Department of Medical Microbiology \& Infectious Diseases, Erasmus University Medical Centre Rotterdam (Erasmus MC), \\ Wytemaweg 80, 3015 CN, Rotterdam, the Netherlands \\ ${ }^{4}$ Department of General Practice, Erasmus University Medical Centre Rotterdam (Erasmus MC), Wytemaweg 80, 3015 CN, \\ Rotterdam, the Netherlands \\ *Author for correspondence: j.hays@erasmusmc.nl
}

General practitioners stand at the front line of healthcare provision and have a pivotal role in the fight against increasing antibiotic resistance. In this respect, targeted antibiotic prescribing by general practitioners would help reduce the unnecessary use of antibiotics, leading to reduced treatment failures, fewer side-effects for patients and a reduction in the (global) spread of antibiotic resistances. Current 'gold standard' antibiotic resistance detection strategies tend to be slow, taking up to $48 \mathrm{~h}$ to obtain a result, although the implementation of point-of-care testing by general practitioners could help achieve the goal of targeted antibiotic prescribing practices. However, deciding on which antibiotic resistances to include in a point-of-care diagnostic is not a trivial task, as outlined in this publication.

First draft submitted: 14 March 2018; Accepted for publication: 10 May 2018; Published online: 16 August 2018

Keywords: antibiotic resistance $\bullet$ general practice $\bullet$ point-of-care diagnostics

The continued spread of antibiotic resistance is a global problem with the WHO predicting 10 million global deaths due to antibiotic resistance by 2050 [1,2]. One way of helping defeat the emergence of antibiotic resistance is via the accurate prescribing of antibiotics only to those patients who are suffering from bacterial (instead of viral) infections. The problem of accurate antibiotic prescribing is especially relevant for general practitioners (GPs) [3,4]. GPs stand on the front line of patient care (primary care), most often being the first point of contact between medical specialists and the general public. However, the rapid differentiation between a bacterial and viral infection in primary care patients is especially difficult to achieve, since the patient's history and physical examination have only a limited value in differentiating between a viral and a bacterial infection [5,6]. Further, if specimens are sent to a centralized diagnostic laboratory for traditional 'gold standard' testing, the results of pathogen isolation, identification and antibiotic resistance detection may take several days to arrive back at the GP practice. Taken together, this means that accurate, evidence-based diagnosis and targeted antibiotic prescribing by GPs are currently problematic. One possible approach to this problem is to implement so called point-of-care (POC) diagnostics at the GP practice and many different POC diagnostics are currently available, or are being developed, to detect infectious diseases [7]. These POC diagnostics can be generally divided into three 'classes': POC diagnostics that differentiate between bacterial and viral infections (without defining which pathogen is present), for example, diagnostics based on inflammatory biomarkers; POC diagnostics that detect and report a specific pathogen that may be present, but (in case of a bacterial infection) do not provide information on the presence of antibiotic resistances, for example, diagnostics based on nucleic acid amplification techniques; and POC diagnostics that detect and report a specific pathogen and the presence of antibiotic resistance, for example, diagnostics based on nucleic acid amplification techniques coupled to microarrays. The ability to accurately differentiate between a viral and bacterial infection may help enormously in reducing the amount of unnecessary antibiotics prescribed to patients suffering from viral infections. This may

Future Medicine 
Table 1. Potential problems and possible solutions to choosing antibiotic resistance targets for incorporation in point-of-care diagnostics for use by general practitioners.

\section{Current problem}

Large diversity in antibiotic resistance mechanisms in primary care

Changing epidemiology of antibiotic resistance due to travel- and ethnic background-associated carriage

Confounding influence and impact of the human microbiota

Lack of knowledge of national antibiotic-prescribing guidelines disadvantages

Will antibiotic resistance diagnostics actually alter GP/patient behavior?

\section{Possible solution}

Development of novel POC diagnostics to detect (combinations of): hydrolyzing enzymes or genes; mutation events; downregulation of efflux pumps; increase or loss of bacterial porins; and metabolically inactive bacterial cells

POC diagnostic innovators ensure that their diagnostics are built around a 'flexible format' to allow for rapid and easy adaptation to new and emerging antibiotic resistances

Continuous epidemiological analysis of the incidence of specific antibiotic resistance genes within primary care and the target market area. This goal could be achieved by ensuring 'connectivity' between (different) POC devices within a single GP practice to the local patient information system

Ultimately, linking connected POC devices within local geographical regions could generate broader (including increased regional) data for GPs and POC developers on the past and current spread and future trends of antibiotic resistance within their (target) region

Acquire knowledge regarding the local incidence of antibiotic resistance and the potential effect of traveler-associated colonization/infection on the prescribing practices of local GPs

Develop POC diagnostics that can distinguish between antibiotic resistances present in pathogens compared with nonpathogens in the human microbiota

Obtain more detailed scientific information on the specific spread of antibiotic resistance between nonpathogens and pathogens - in order to provide advice on whether an antibiotic resistance gene in a nonpathogenic species is likely to spread to a pathogenic species during antibiotic treatment; and how this information should affect antibiotic prescribing practices and guidelines

Take note of antibiotic-prescribing guidelines in different countries (may indicate current/future resistance trends). Be aware that these guidelines may change regularly

POC diagnostics innovators ensure that their POC diagnostics are 'flexible' for rapid and easy adaptation to changes in national antibiotic-prescribing guidelines

Be aware of potential limitations associated with the molecular detection of antibiotic resistance genes. Gene promoter and coding region sequencing may provide some idea of the expression of specific antibiotic resistance genes, but mRNA or protein-based diagnostics may be required. Note that even mRNA and protein may not detect 'dormant' antibiotic resistance genes that have not yet been exposed to antibiotics

The development of a rapid phenotypic detection test would act as a 'catch-all' technique, providing a simple answer to the question of antibiotic resistance for many antibiotic resistance mechanisms (mobile genetic elements, mutations, efflux, porins, etc.). Note that this may not be true for 'metabolically inactive, but antibiotic resistant' microorganisms

The factors influencing the prescribing of antibiotics, e.g., time-to-result, costs, added value, etc., by GPs need to be better understood and factored into POC diagnostic development

GP: General practitioner; POC: Point-of-care.

in part be calculated via POC-assisted measurement of nonspecific biomarkers of infection, for example, C-reactive protein (CRP) and procalcitonin (PCT), though nonspecific biomarkers do not indicate if a bacterial pathogen is resistant to a particular antibiotic or not $[8,9]$. Further, if a bacterial infection is indicated or detected, there is still a chance that GPs may prescribe an antibiotic that is not suitable for that infection due to a lack of information on the possible expression of antibiotic resistance by the causative bacterial pathogen. Therefore, in cases where a bacterial infection is established, it could be potentially useful if GPs were also able to determine not only the presence of bacterial or viral pathogens, but also the presence of antibiotic resistances. In practice, however, the actual choice and feasibility of developing antibiotic resistance detection panels for use in POC diagnostics in primary care may be more difficult than is generally appreciated by POC innovators, microbiologists and GPs. In this article, the authors highlight some of the major challenges and barriers that currently exist in choosing antibiotic resistance targets for use in POC diagnostics by GPs (Table 1). The material presented is based on discussions that took place as part of the EU-funded H2020 Diagoras project (www.diagoras.eu), which intends to develop a POC diagnostic device for use by GPs and dentists. 


\section{Diversity in antibiotic resistance mechanisms}

The type of antibiotic resistance and antibiotic resistance mechanisms found in the primary care setting depends on several factors, including the availability and use of antibiotics by GPs and patients within a particular geographical location and community. Therefore, the types of resistances to be detected may be very diverse, including both intrinsic and acquired resistances. Intrinsic resistance is an inherent characteristic of microorganisms that makes them insensitive to particular antibiotics, with an example being the inherent resistance of Gram-negative microorganisms to the antibiotic vancomycin due to the inability of vancomycin to efficiently penetrate the Gram-negative bacterial outer membrane. Acquired antimicrobial resistance is, however, most relevant for antimicrobial prescribing in healthcare as they can generate resistance in previously nonintrinsically resistant (antibiotic susceptible) microorganisms. Further, this resistance can be readily transferred between different bacterial species (either in the presence or absence of antibiotics). Transferable antibiotic resistance genes may be present in different mobile genetic elements (MGE), carried for example in many different types of plasmids (via conjugation), or transferred by the uptake of naked DNA (transfection), or spread via bacteriophage-mediated transfer (transduction). Examples of antimicrobial resistance genes carried by MGE include genes coding for $\beta$-lactam-based antibiotic hydrolyzing proteins, such as extended spectrum $\beta$-lactamases (ESBL) and carbapenemases, for example, Klebsiella pneumoniae carbapenemase, Verona integron-encoded metallo- $\beta$ lactamase, etc. Further, for each gene there may be many different variants, for example, $>160$ TEM (isolated from patient Temoneira) and >100 sulfhydryl variant (SHV) $\beta$-lactamase gene variants [10]. Antibiotic resistance may also originate via nontransferable genetic events, that is, genetic mutations. For example, mutations in the quinolone resistance-determining region of the gyrase $\mathrm{A}(\mathrm{gyr} A)$ gene may result in bacterial resistance to quinolone-based antibiotics such as ciprofloxacin and nalidixic acid. Genetic mutations may also be associated with the downregulation of porins or the upregulation of efflux pumps [11,12]. Further, many different resistance mechanisms may be present within a single isolate of a bacterium at the same time, resulting in multiresistant or even pan-resistant microorganisms [13,14]. With particular respect to general practice, and as an example, community respiratory tract infections in the Netherlands are generally treated using amoxicillin and as a second or third choice tetracyclines and macrolides, while urinary tract infections are generally treated using nitrofurantoin, phosphomycin, trimethoprim and ciprofloxacin. Just some of the antibiotic resistances associated with these combinations of antibiotics include hyper production of penicillinases, plasmidal resistances, point mutations, efflux pumps, etc. [15-17]. Such a diversity of antimicrobial resistance mechanisms means that it is unlikely (or feasible) to develop a POC diagnostic that can detect all of the different antibiotic resistances described in the scientific literature. Instead, a 'targeted' approach may be required, whereby diagnostics are targeted to the antimicrobial resistance actually circulating within a given geographical location or community. This solution, however, generates two problems: diagnostic innovators need to have access to up-to-date epidemiological data on antibiotic resistances for their target healthcare settings; and the existence of novel antimicrobial resistances, or the introduction of antimicrobial resistances from neighboring geographical locations or communities, may be missed. Further, if POC diagnostic innovators include many tens of antibiotic resistances in their detection panels, will GPs be knowledgeable enough about the significance of the different resistances for their patients? Will the relevant POC diagnostic therefore have to provide advice to the GP, dependent on the antibiotic-resistant results? Giving such advice could be seen as advising the GP how to treat the patient, rather than the GP making his own decision. This may have legal and ethical implications. Finally, as the number of POC diagnostic antibiotic resistance targets increases, so does the price of the diagnostic, which may negatively affect the value: cost ratio of the diagnostic. GPs may then perhaps ask themselves why they need to buy a diagnostic that detects many different antibiotic resistances that may not be relevant to their own geographical location or community.

\section{Local incidence of antibiotic resistance genes}

The incidence of antibiotic resistance genes may vary per GP geographic location and in time, making it difficult for innovators to predict future trends in antibiotic resistance profiles. For example, $\beta$-lactam resistance in Haemophilus influenzae (associated with respiratory tract infections in primary care) has previously been associated with the carriage and expression of the transferable rob-1 $\beta$-lactamase genes [18]. However, there appear to be differences in the carriage of rob-1 between Canada, USA, Mexico, Scotland and Turkey [19-21]. That said, the most likely antibiotic resistance phenotype associated with a specific disease in a particular geographical location or community may already be known by the GP via his own empirical experience of recent treatment successes and failures. This empirical knowledge (along with national guidelines) may mean that antibiotic resistance testing is seen as unnecessary by some GPs. Additionally, if a POC diagnostic is implemented, which determines bacterial 
identification only, the GP may already be empirically aware of the most likely antibiotic resistances associated with that particular bacterial pathogen. For example, the bacterium Moraxella catarrhalis is associated with upper respiratory tract infections such as otitis media in children and lower respiratory tract infections such as exacerbations of chronic obstructive pulmonary disease in adults [22-24]. This bacterial pathogen is associated with the carriage of a single unique $\beta$-lactamase enzyme, which can be present in one of two slightly different forms (Bro- 1 and Bro-2) [25]. Further, epidemiological studies have shown that penicillin resistance and the carriage of bro genes approach $90 \%$ in clinical isolates, meaning that a POC diagnostic that detects the bro gene may not actually be useful (or seen as financially viable) to GPs, especially when a broad-spectrum alternative such as erythromycin is available for prescription. Of course, erythromycin resistance in clinical isolates of $M$. catarrhalis is known, but the incidence of such resistance is relatively low in most regions of the world [26].

\section{Travel- \& community-based antibiotic resistance}

Possibly influencing the range of antibiotic resistance genes seen by a GP within his/her practice is the fact that travelers (including holidaymakers, sales representatives, etc.), as well as patients undergoing surgery (including elective cosmetic surgery) may have recently visited countries with a high prevalence of antibiotic resistance, and subsequently become colonized with nonlocal antibiotic-resistant bacteria during their travels [27]. Examples of such nonlocal antibiotic resistances could include various ESBL [28] and New Delhi $\beta$-lactamase-1 (NDM1) producing bacteria [29]. Additionally, communities containing relatively high levels of citizens from ethnic minority backgrounds may also show differences in the range of antibiotic resistances carried [30]. The question then for POC diagnostic innovators is 'how relevant are travel- and ethnic background-related antibiotic resistance in patients attending GP practices in different geographical locations?' Unfortunately, the specific collection of travel- and community-based antibiotic resistance data is likely to be too expensive for most POC diagnostic innovators.

Important also to remember, is that once GPs start using 'stronger and more unusual' antibiotics against pathogens that have become resistant to the 'standard antibiotics' prescribed within general practice, the cryptic (asymptomatic) carriage of more unusual 'travel/ethnic background-associated' antibiotic resistances within the community setting could become important. In this scenario, the vertical transmission of such genes to related/unrelated bacterial species could also impact on the risk of infection by pathogens already resistant to previously used antibiotics. This in turn, could mean that, by the time POC developers place a diagnostic on the healthcare market (approximately 10 years), the antibiotic resistance problem may have changed from their original data.

\section{The human microbiota}

The detection of an antibiotic resistance gene within a clinical sample does not automatically mean that the causative bacterial pathogen of an illness actually carries the resistance gene detected. This is especially true for transferable genes present on MGE, where identical antibiotic resistance genes can be widely spread, not only within bacterial pathogens, but also within the normal human microbiota of the patient [31]. If an antibiotic resistance gene is found to be present in the clinical sample, how can the clinician be sure that the antibiotic resistance gene is present within the bacterial pathogen actually causing the disease and not present in a 'harmless' member of the patient's microbiota? [32-34]. Focusing on the detection of MGE-associated antibiotic resistance genes may partially get around the problem associated with the presence of antibiotic resistance genes within the human microbiota, as these are likely to be able to be transferred to pathogenic bacteria. Additionally, the GP may have to take note of any recent exposure of the patient to antibiotics, as antibiotic resistance generated by previous exposure to antibiotics could persist within the patient's human microbiota for some time [35-39]. Note also that as more antibiotic resistance targets are included in a POC diagnostic, the chance of detecting antibiotic resistance genes in the normal human microbiota (including nonpathogenic microorganisms) of the patient will also increase, possibly yielding 'false positive' results - at least with respect to the antibiotic sensitivity of the actual pathogen [40].

\section{National guidelines \& market perspectives}

Many countries produce national guidelines for the treatment of diseases often seen within primary care. Examples of such guidelines include the National Institute for Health and Care Excellence (NICE, UK) and The Nederlands Huisartsen Genoostchap (NHG, The Netherlands) [41,42]. It is essential that POC diagnostic innovators who are thinking of developing antibiotic resistance diagnostics actually take note of these guidelines and develop tests for antimicrobial resistances that are based on the antibiotic-prescribing guidelines of the countries in which the 
device is to be sold. However, for the POC diagnostic innovator, developing different antibiotic resistance testing capacities for specific countries may not make financial sense unless those countries have standardized primary care guidelines available and large populations with access to primary care. Additionally, national guidelines for antibiotic prescribing are regularly revised, which means that POC diagnostic innovators must consider if the time required to develop, market and generate profit from a new antibiotic resistance test will fit within the regular cycle of national guideline revision. Of course, the revision of national guidelines does not mean to say that the actual choice of antibiotics to be used will be changed, but the POC diagnostic innovator should take this into consideration when weighing up the time from POC development to marketing. Finally, if a GP knows that a revision of antibiotic-prescribing guidelines is due in a few months, he may be less inclined to buy an antibiotic resistance diagnostic that may be 'out of date' within a few months. One possible solution to this problem is for POC diagnostic innovators to be aware of the problem and develop diagnostics that are easily and quickly adaptable to the rapidly changing 'ecosystem' of (inter)national guidelines and to the emergence of new antibiotic resistances.

\section{Molecular versus phenotypic analysis}

Phenotypic analysis is a gold standard 'catch-all' technique that determines antibiotic resistance via the inability of a microorganism to replicate in the presence of a minimum inhibitory concentration (MIC) of an antibiotic. However, current problems with phenotypic detection include the 'time to result', sensitivity and the general need for centralized laboratory testing compared with nucleic acid amplification techniques [43]. Additionally, the majority of currently available POC infectious disease diagnostics rely on nucleic acid-based amplification (usually involving polymerase chain reaction (PCR) or isothermal amplification techniques) [44]. The detection of MGE-associated antibiotic resistance genes by nucleic amplification techniques, using conserved regions of DNA as targets, is highly suited to the detection of many (transferable) antibiotic resistance genes. However, antibiotic resistance that occurs due to genetic mutations are less easily detected using standard nucleic acid amplification techniques. This is because these mutations mostly occur in genes normally present in the pathogenic bacterium, for example, ciprofloxacin resistance and the quinolone-resistance determining region, and the detection of the gene per se provides no information on actual phenotypic antibiotic resistance. However, POC diagnostics that detect mutations in combination with gene amplification, for example, gene amplification followed by microarray detection, or gene sequencing [45], may have a distinct advantage over gene amplification-only POC diagnostics with respect to mutation-associated antibiotic resistance. Essentially, gene amplification-only diagnostics could be perceived as not offering the GP enough clinical information (antibiotic resistance caused by mutations) on which to base an informed antibiotic-prescribing decision. This could seriously impact on the perceived value of a nucleic acid amplification-only diagnostic device to the GP.

\section{Will antibiotic resistance POC diagnostics actually alter GP/patient behavior?}

Finally, another unanswered question is whether the availability of POC antibiotic resistance data to GPs will actually alter their antibiotic-prescribing practices. GPs may prescribe antibiotics 'just in case' there is a chance of a bacterial infection (even though an available POC diagnostic has already shown the presence of a viral infection). The reasoning here is that an undetected bacterial superinfection may occur on top of the viral infection. Another potential unanswered question is 'how will GPs behave in their antibiotic-prescribing practices when a POC diagnostic device determines the existence of both a viral and bacterial infection? A GP's clinical decision-making will probably depend on the patient's physical examination and the type of virus (influenza virus or coronavirus, for example) and the type of bacterium detected. Interestingly, however, the introduction of antibiotic resistance testing by GPs may help 'persuade' patients that a decision not to prescribe antibiotics is 'rational' and based on scientific testing [46].

\section{Conclusion and Future perspective}

Choosing useful antibiotic resistance gene targets for use in POC diagnostics by general practitioners is not an easy task. Currently, the choice is particularly limited by: the diversity of antibiotic resistance mechanisms present in general practice; a lack of epidemiological information on the origin and spread of antibiotic resistance mechanisms within particular geographical locations and within particular communities; and limitations on currently available POC diagnostic technologies to rapidly detect the many different types of antibiotic resistance mechanisms known. Lesser problems include: the inaccuracy of predicting future trends in antibiotic resistance; and a lack of knowledge of the impact of the human microbiota on antibiotic resistance carriage and antibiotic treatment failure. It should 
be noted that, even if the above challenges are successfully overcome, the success or failure of the implementation of future POC antibiotic resistance diagnostics into general practice depends on many interacting factors. These factors include the behavioral decision-making process of individual general practitioners, time-to-result and the (perceived) added value of implementing POC antibiotic resistance diagnostics into the GP practice.

\section{Executive summary}

- General practitioners are the first line of defense against antibiotic resistance within communities.

- Better targeted antibiotic prescribing, for example, by the use of point-of-care (POC) diagnostics could potentially help reduce the amount of unnecessary antibiotics prescribed to patients.

- The choice of antibiotic resistance targets for POC diagnostic development is currently problematic.

- Antibiotic resistance mechanisms in general practice are diverse.

- Knowledge of the incidence of specific antibiotic resistance mechanisms is required in order to generate guidelines that indicate if a specific POC diagnostic is actually clinically relevant.

- Scientific information may be required regarding the specific incidence of antibiotic resistance between nonpathogens and pathogens.

- POC diagnostic innovators should take note of the antibiotic-prescribing guidelines of different countries (may indicate future resistance trends).

- The range and incidence of antibiotic resistance present in a community could be affected by travelers (holidaymakers and/or healthcare migrants, for example, patients who travel abroad for cosmetic surgery procedures) and by the ethnic background of the local population.

- Be aware of potential limitations associated with the molecular detection of antibiotic resistance genes by POC diagnostics.

- The development of a rapid phenotypic detection test would act as a 'catch-all' technique, providing a simple answer to the question of antibiotic resistance for most antibiotic resistance mechanisms.

Financial \& competing interests disclosure

This publication was supported by a Horizon2020 EU grant under grant agreement GA-633780 ('DIAGORAS' - www. diagoras.eu). The authors have no other relevant affiliations or financial involvement with any organization or entity with a financial interest in or financial conflict with the subject matter or materials discussed in the manuscript apart from those disclosed.

No writing assistance was utilized in the production of this manuscript.

Open access

This work is licensed under the Attribution-NonCommercial-NoDerivatives 4.0 Unported License. To view a copy of this license, visit http://creativecommons.org/licenses/by-nc-nd/4.0/

\section{References}

Papers of special note have been highlighted as: $\bullet$ of interest; $\bullet \bullet$ of considerable interest

1. WHO. United Nations meeting on antimicrobial resistance (2016). www.who.int/bulletin/volumes/94/9/16-020916.pdf

$\bullet \quad$ Highlights the current and future problem of worldwide antibiotic resistance.

2. de Kraker ME, Stewardson AJ, Harbarth S. Will 10 million people die a year due to antimicrobial resistance by 2050? PLoS Med. 13(11), e1002184 (2016).

3. Kaman WE, Elshout G, Bindels PJ, Mitsakakis K, Hays JP. Current problems associated with the microbiological point-of-care testing of respiratory tract infections in primary care. Future Microbiol. 11, 607-610 (2016).

- Highlights some general problems associated with the implementation of infectious disease point-of-care diagnostic devices into general practice.

4. Kuehlein T, Szecsenyi J, Gutscher A, Laux G. Antibiotic prescribing in general practice - the rhythm of the week: a cross-sectional study. J. Antimicrob. Chemother. 65(12), 2666-2668 (2010).

5. Hopstaken RM, Stobberingh EE, Knottnerus JA et al. Clinical items not helpful in differentiating viral from bacterial lower respiratory tract infections in general practice. J. Clin. Epidemiol. 58(2), 175-183 (2005).

6. Shaikh N, Swaminathan N, Hooper EG. Accuracy and precision of the signs and symptoms of streptococcal pharyngitis in children: a systematic review. J. Pediatr. 160(3), 487.e483-493.e483 (2012).

7. Zumla A, Al-Tawfiq JA, Enne VI et al. Rapid point of care diagnostic tests for viral and bacterial respiratory tract infections - needs, advances, and future prospects. Lancet Infect. Dis. 14(11), 1123-1135 (2014). 
8. van der Meer V, Neven AK, van den Broek PJ, Assendelft WJ. Diagnostic value of C reactive protein in infections of the lower respiratory tract: systematic review. BMJ 331(7507), 26 (2005).

9. Aabenhus R, Jensen JU, Jorgensen KJ, Hrobjartsson A, Bjerrum L. Biomarkers as point-of-care tests to guide prescription of antibiotics in patients with acute respiratory infections in primary care. Cochrane Database Syst. Rev. (11), CD010130 (2014).

10. The Lactamase Engineering Database. www.laced.uni-stuttgart.de/

- Details the genetic diversity of $\beta$-lactam genes.

11. Blair JM, Webber MA, Baylay AJ, Ogbolu DO, Piddock LJ. Molecular mechanisms of antibiotic resistance. Nat. Rev. Microbiol. 13(1), 42-51 (2015).

12. van Hoek AH, Mevius D, Guerra B, Mullany P, Roberts AP, Aarts HJ. Acquired antibiotic resistance genes: an overview. Front. Microbiol. 2, 203 (2011).

-• Details the diversity of antibiotic resistance mechanisms.

13. Batard E, Lefebvre M, Aubin GG, Caroff N, Corvec S. High prevalence of cross-resistance to fluoroquinolone and cotrimoxazole in tetracycline-resistant Escherichia coli human clinical isolates. J. Chemother. 28(6), 510-512 (2016).

14. Chen L, Todd R, Kiehlbauch J, Walters M, Kallen A. Notes from the field: pan-resistant New Delhi metallo- $\beta$-lactamase-producing Klebsiella pneumoniae - Washoe County, Nevada, 2016. MMWR Morb. Mortal. Wkly Rep. 66(1), 33 (2017).

15. Ortega A, Oteo J, Aranzamendi-Zaldumbide M et al. Spanish multicenter study of the epidemiology and mechanisms of amoxicillin-clavulanate resistance in Escherichia coli. Antimicrob. Agents Chemother. 56(7), 3576-3581 (2012).

16. Yang HJ, Song DJ, Shim JY. Mechanism of resistance acquisition and treatment of macrolide-resistant Mycoplasma pneumoniae pneumonia in children. Korean J. Pediatr. 60(6), 167-174 (2017).

17. Zeller V, Janoir C, Kitzis MD, Gutmann L, Moreau NJ. Active efflux as a mechanism of resistance to ciprofloxacin in Streptococcus pneumoniae. Antimicrob. Agents Chemother. 41(9), 1973-1978 (1997).

18. Shanahan PM, Thomson CJ, Amyes SG. Antibiotic susceptibilities of Haemophilus influenzae in central Scotland. Clin. Microbiol. Infect. 1(3), 168-174 (1996).

19. Kuvat N, Nazik H, Berkiten R, Ongen B. Tem-1 and Rob-1 presence and antimicrobial resistance in haemophilus influenzae strains, Istanbul, Turkey. Southeast Asian J. Trop. Med. Public Health 46(2), 254-261 (2015).

20. Farrell DJ, Morrissey I, Bakker S, Buckridge S, Felmingham D. Global distribution of TEM-1 and ROB-1 $\beta$-lactamases in Haemophilus influenzae. J. Antimicrob. Chemother. 56(4), 773-776 (2005).

21. Karlowsky JA, Verma G, Zhanel GG, Hoban DJ. Presence of ROB-1 $\beta$-lactamase correlates with cefaclor resistance among recent isolates of Haemophilus influenzae. J. Antimicrob. Chemother. 45(6), 871-875 (2000).

22. Wald ER. Acute otitis media and acute bacterial sinusitis. Clin. Infect. Dis. 52(Suppl. 4), S277-S283 (2011).

23. Sillanpaa S, Oikarinen S, Sipila M et al. Moraxella catarrhalis might be more common than expected in acute otitis media in young Finnish children. J. Clin. Microbiol. 54(9), 2373-2379 (2016).

24. Perez AC, Murphy TF. Potential impact of a Moraxella catarrhalis vaccine in COPD. Vaccinedoi:10.1016/j.vaccine.2016.12.066 (2017) (Epub ahead of print).

25. Eliasson I, Kamme C, Vang M, Waley SG. Characterization of cell-bound papain-soluble $\beta$-lactamases in BRO-1 and BRO-2 producing strains of Moraxella (Branhamella) catarrhalis and Moraxella nonliquefaciens. Eur. J. Clin. Microbiol. Infect Dis. 11(4), 313-321 (1992).

26. Flamm RK, Sader HS, Farrell DJ, Jones RN. Macrolide and tetracycline resistance among Moraxella catarrhalis isolates from 2009 to 2011. Diagn. Microbiol. Infect. Dis. 74(2), 198-200 (2012).

27. Wiklund S, Fagerberg I, Ortqvist A et al. Knowledge and understanding of antibiotic resistance and the risk of becoming a carrier when travelling abroad: a qualitative study of Swedish travellers. Scand. J. Public Health 43(3), 302-308 (2015).

28. Arcilla MS, van Hattem JM, Haverkate MR et al. Import and spread of extended-spectrum $\beta$-lactamase-producing Enterobacteriaceae by international travellers (COMBAT study): a prospective, multicentre cohort study. Lancet Infect. Dis. 17(1), 78-85 (2017).

- Indicates the carriage of newly acquired antibiotic-resistant bacteria after travel.

29. Fernando GA, Collignon PJ, Bell JM. A risk for returned travellers: the 'post-antibiotic era'. Med. J. Aust. 193(1), 59 (2010).

30. Wickramasinghe NH, Xu L, Eustace A, Shabir S, Saluja T, Hawkey PM. High community faecal carriage rates of CTX-M ESBL-producing Escherichia coli in a specific population group in Birmingham, UK. J. Antimicrob. Chemother. 67(5), 1108-1113 (2012).

- Outlines how the carriage of antibiotic-resistant bacteria may vary between specific population groups.

31. Moore AM, Patel S, Forsberg KJ et al. Pediatric fecal microbiota harbor diverse and novel antibiotic resistance genes. PLoS ONE 8(11), e78822 (2013).

32. Shoemaker NB, Vlamakis H, Hayes K, Salyers AA. Evidence for extensive resistance gene transfer among Bacteroides spp. and among Bacteroides and other genera in the human colon. Appl. Environ. Microbiol. 67(2), 561-568 (2001).

33. Sommer MO, Church GM, Dantas G. The human microbiome harbors a diverse reservoir of antibiotic resistance genes. Virulence 1(4), 299-303 (2010). 
- Indicates that the human microbiota is a reservoir for antibiotic resistance genes.

34. Potron A, Poirel L, Nordmann P. Origin of OXA-181, an emerging carbapenem-hydrolyzing oxacillinase, as a chromosomal gene in Shewanella xiamenensis. Antimicrob. Agents Chemother. 55(9), 4405-4407 (2011).

35. Costelloe C, Metcalfe C, Lovering A, Mant D, Hay AD. Effect of antibiotic prescribing in primary care on antimicrobial resistance in individual patients: systematic review and meta-analysis. BMJ 340, c2096 (2010).

36. Jakobsson HE, Jernberg C, Andersson AF, Sjolund-Karlsson M, Jansson JK, Engstrand L. Short-term antibiotic treatment has differing long-term impacts on the human throat and gut microbiome. PLoS ONE 5(3), e9836 (2010).

37. Nys S, Tjhie JH, Bartelds AI, Heijnen ML, Peeters MF, Stobberingh EE. Erythromycin resistance in the commensal throat flora of patients visiting the general practitioner: a reservoir for resistance genes for potential pathogenic bacteria. Int. J. Antimicrob. Agents 26(2), 133-137 (2005).

-• Indicates that commensal bacteria may act as a reservoir for antibiotic resistance genes.

38. Penders J, Stobberingh EE, Savelkoul PH, Wolffs PF. The human microbiome as a reservoir of antimicrobial resistance. Front. Microbiol. 4, 87 (2013).

39. Wang N, Hang X, Zhang M, Liu X, Yang H. Analysis of newly detected tetracycline resistance genes and their flanking sequences in human intestinal bifidobacteria. Sci. Rep. 7(1), 6267 (2017).

40. Hu Y, Yang X, Qin J et al. Metagenome-wide analysis of antibiotic resistance genes in a large cohort of human gut microbiota. Nat. Commun. 4, 2151 (2013).

41. NICE. Respiratory Tract Infections (Self-Limiting): Prescribing Antibiotics. Clinical Guideline [CG69] (2008). www.nice.org.uk/guidance/cg69

42. NHG Standaard Acuut hoesten [Dutch General Practitioner Society Standard - Acute Coughing] (2011). www.nhg.org/standaarden/volledig/nhg-standaard-acuut-hoesten

43. Maurer FP, Christner M, Hentschke M, Rohde H. Advances in rapid identification and susceptibility testing of bacteria in the clinical microbiology laboratory: implications for patient care and antimicrobial stewardship programs. Infect. Dis. Rep. 9(1), 6839 (2017).

44. Craw P, Balachandran W. Isothermal nucleic acid amplification technologies for point-of-care diagnostics: a critical review. Lab. Chip. 12(14), 2469-2486 (2012)

45. Zankari E, Allesoe R, Joensen KG, Cavaco LM, Lund O, Aarestrup FM. PointFinder: a novel web tool for WGS-based detection of antimicrobial resistance associated with chromosomal point mutations in bacterial pathogens. J. Antimicrob. Chemother. 72(10), 2764-2768 (2017).

46. Butler CC, Simpson S, Wood F. General practitioners' perceptions of introducing near-patient testing for common infections into routine primary care: a qualitative study. Scand. J. Prim. Health Care 26, 17-21 (2008). 